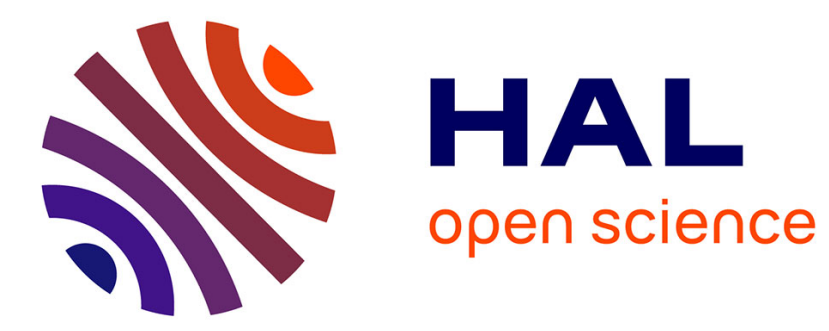

\title{
On the semantics of English coordinate compounds Vincent Renner
}

\section{To cite this version:}

Vincent Renner. On the semantics of English coordinate compounds. English Studies, 2008, 89 (5), pp.606-613. hal-00512305

\section{HAL Id: hal-00512305 \\ https://hal.science/hal-00512305}

Submitted on 29 Aug 2010

HAL is a multi-disciplinary open access archive for the deposit and dissemination of scientific research documents, whether they are published or not. The documents may come from teaching and research institutions in France or abroad, or from public or private research centers.
L'archive ouverte pluridisciplinaire HAL, est destinée au dépôt et à la diffusion de documents scientifiques de niveau recherche, publiés ou non, émanant des établissements d'enseignement et de recherche français ou étrangers, des laboratoires publics ou privés. 


\section{On The Semantics Of English Coordinate Compounds}

\section{Vincent Renner}

\section{Introduction}

In the current literature on English compounding, the most common terms to designate those compounds whose internal semantic relation is coordinate (ex.: space-time, bittersweet, st $\left.{ }^{`} p-g^{`}\right)$ are dvandva and $c^{`}$ pulative c`mp`und. ${ }^{`}$ Several morphologists - e.g. ten Hacken, Plag, and Katamba and Stonham ${ }^{2}-$ consider that the two terms are synonymous, and they use them indiscriminately. Dvandva is however an ambiguous term as other linguists - e.g. Arnaud and Bauer ${ }^{3}-$ use it only in the case of heteroreferential coordinate compounding, i.e. for compounds composed of two nouns whose denotata are unfused (ex: dinner dance, tract $r$-trailer). They do so in accordance with the historical meaning of the term, which was originally used by Sanskrit grammarians to designate heteroreferential coordinate compounds. ${ }^{4}$ This ambiguity sometimes leads to confusion, as the following remark by $\mathrm{Plag}^{5}$ shows: “It is `ften stated that dvandva c` $m p$ `unds are $n^{`} t$ very c`mm` $n$ in English (e.g. Bauer 1983:203), but in a m`re recent study by Olsen (2001) hundreds `f attested $f^{`}$ rms are listed, which sh`ws that such c`mp`unds are far fr` $m$ marginal.” When using dvandva, Bauer refers to non-embedded heteroreferential compounds (ex.: Alsace-L 'rraine), whereas Olsen's corpus contains almost exclusively either homoreferential or embedded heteroreferential compounds (ex.: dancer-singer; patient-d ct $^{`} r$ [partnership]). The extension of the concept DVANDVA sometimes includes compounds whose elements are not simply juxtaposed. Bauer ${ }^{6}$ applies the term to syndetic coordinate compounds (ex.: bubble-and-squeak, milk-and-water), even though he stresses that these compounds differ from true dvandvas because a coordinator is inserted

\footnotetext{
${ }^{1}$ I am grateful to Pierre Arnaud, Nicolas Ballier, Laurie Bauer, and Diana Lewis for helpful comments on earlier versions of this research. Errors are my own.

${ }^{2}$ Ten Hacken, 122; Plag, 146; Katamba and Stonham, 333.

${ }^{3}$ Arnaud, 4; Bauer, Gl`ssary ‘f $M$ `rph`l’gy, 41.

${ }^{4}$ Cf. Olsen, 281-4.

${ }^{5}$ Plag, 147.

${ }^{6}$ Bauer, English W`rd-F'rmati n, 207.
} 
between the two nouns. Boisson ${ }^{7}$ does the same, and he even adds a blend, Oxbridge $(<$ Oxf $r d$ + Cambridge), to his list of nominal dvandva compounds.

Coordinate compounds such as player-manager, pale-dry, and $c^{\prime}{ }^{\prime} k$-chill do not have pride of place in the literature on English compounding. This is due to the fact that such compounds are infrequent - Arnaud $^{8}$ estimates for instance that $98 \%$ of the nominal compounds listed in the COD10 are subordinate, which means implicitly that coordinate compounds count for no more than $2 \% .^{9}$ It is unfortunate that this relative numerical paucity hides the fact that these compounds are highly remarkable from a semantic standpoint, and the present article aims to offer new insight on their behaviour, which is far from uniform, and gives rise to a complex typology.

\section{Definition}

A compound is coordinate only if it is composed of two elements that belong to the same lexical category and are co-hyponyms. These criteria are however not sufficient to determine the coordinate status of a compound, and the use of a definitional test-sentence is necessary. The first one that comes to mind serves to verify that the $X . Y^{10}$ compound corresponds to a coordinate paraphrase. The test "(an) $X . Y$ is (an) $X$ and (a) $Y$ ” is passed by a large number of noun-noun compounds that are intuitively coordinate, but it is in some cases negative for compounds that are non-subordinate, such as $w^{`}$ lf $d^{`} g^{11}$ (*a $w^{`}$ lf $d^{\prime} g$ is a $w^{`}$ lf and a d`g) and $s^{`}$ utheast $\left({ }^{*} s^{`}\right.$ utheast is $s^{`}$ uth and east). In addition, the test is positive for compounds such as carrier pige` $n$ and manservant, even though the first noun is clearly subordinate to the second one (the compounding elements are not co-hyponyms; the first noun is used to mark function or gender). The same problem arises with adjective-adjective and verb-verb compounds: the test " $t$ " be $X . Y$ is $t$ ' be $X$ and

\footnotetext{
${ }^{7}$ Boisson, 427-8, 573, 661.

${ }^{8}$ Arnaud, 4.

${ }^{9}$ This observation is not universal: coordinate compounds are common in many languages from Continental East and South-East Asia (Wälchli, 215).

${ }^{10}$ It is an acknowledged fact that the spelling of English compounds is erratic. X.Y is therefore used as a convention to refer indiscriminately to continuous compounds $(X Y)$, hyphenated compounds $(X-Y)$, and discontinuous compounds $(X Y)$.

${ }^{11} W^{\prime}$ If $d ` g$ refers here to a cross between a wolf and a domestic dog.
} 
$Y^{\prime \prime}$ is not fully adequate for A.A compounds as a subordinate compound like red- $h^{`} t$ passes it while blue-green does not, even though its definition (“A c`l` $r$ ab`ut midway between blue and green in the spectrum." RHUD) shows it is not subordinate; the test " $t$ ' $X . Y$ is $t^{\prime} X$ and $t^{\prime} Y^{\prime}$ " is in the same way not discriminating as it is positive for almost any V.V compound, even though verbs like $b l^{`} w$ dry and crash-land, which are made of a taxonym and its hypernym, are not coordinate, the first verb being a marker of manner that is subordinate to the second one (bl`wing s`mething is a way ` $f$ drying it; crashing s`mething is a way `f landing it $).{ }^{12}$

Coordination refers to same-level ordering, which means in the present case that there is no dependency of one compounding element upon the other. It is therefore natural to assume that internal word order is somewhat arbitrary in coordinate compounds whereas it is strictly constrained in subordinate compounds. This leads to the formulation of a metalinguistic test applicable to all lexical categories: "the c`mp`und X.Y c`uld be named Y.X”. This approach is superior to the previous one as it does justice to the intuitions remarked above: $d^{\prime} g w^{`} l f$, easts`uth, and green-blue are acceptable forms, but pige` $n$ carrier, $h^{`} t$-red, dry-bl` $w$, and land-crash are counterintuitive to denote the concepts associated with the corresponding $X . Y$ forms. Passing the reversibility test is therefore a sufficient criterion to determine the coordinate status of a compound; the test is however not ideal, as it does not work with coordinate compounds whose elements denote asynchronous events or actions, such as murder-suicide ${ }^{13}$ and freeze-dry.

\section{Noun-noun compounds}

In view of the data, three major semantic types of N.N coordinate compounds need to be distinguished, each one corresponding to a specific test-sentence:

i. multifunctionality test: “(an) X.Y is (an) Xwh /which is als` (a) Y”

ii. additionality test: "(an) X.Y is (an) X plus (a) Y”

\footnotetext{
${ }^{12}$ The terms and the test are borrowed from Cruse, 139.

${ }^{13}$ This compound is bisemic: it may either refer to a suicide-bombing, in which case the two events are synchronous, or to a murder followed by the suicide of the offender, in which case the two events are asynchronous.
} 
iii. hybridity test: “(an) X.Y is ab` ut midway between (an) X and (a) $Y$ ”,14

This ternary division is interestingly echoed by the arrangement in Table 1, in which two features of any coordinate compound are cross-tabulated, i.e. its semantic headedness and the referential relation between the denotata of the compounding elements. Multifunctional compounds are doubly endocentric, each element being a semantic head of the whole (ex.: a hunter-gatherer is a hunter; a hunter-gatherer is a gatherer), whereas additional and hybrid compounds are exocentric (ex.: *a

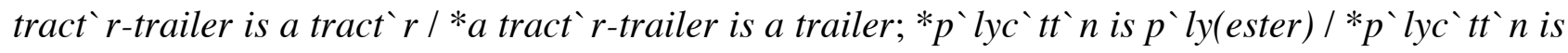
$\left.c^{`} t t^{`} n\right)$. Multifunctional and hybrid compounds denote entities formed by the fusion of the denotata of the compounding elements whereas additional compounds denote entities formed by the juxtaposition of the denotata.

\section{Table 1}

Cross-tabulation of the features semantic headedness and referential relati $n$ on the class of N.N coordinate compounds

\begin{tabular}{|c|c|c|}
\hline & endocentric & exocentric \\
\hline homoreferential & $\begin{array}{c}\text { multifunctional } \\
\text { (hunter-gatherer) }\end{array}$ & $\begin{array}{c}\text { hybrid } \\
\left(p^{`} l y c^{`} t t^{`} n\right)\end{array}$ \\
\hline heteroreferential & - & $\begin{array}{c}\text { additional } \\
\text { (tract' } r \text {-trailer) }\end{array}$ \\
\hline
\end{tabular}

Multifunctional compounds denote mainly individuals (ex.: hunter-gatherer, 'wner-`ccupier, player-manager, student-athlete) and artifacts (ex.: fighter-b`mber, hammer-axe, s`fa bed), additional compounds mainly artifacts (ex.: camiknickers, fridge-freezer, penny-farthing, tract $r$ trailer), substances (ex.: gum resin, $p^{`} l y c^{`} t t^{`} n$, tarmacadam, $t^{`} x$ in-antit $x i n$ ), and animate beings (ex.: ape-man, bear cat, bull terrier, bullmastiff, tr` utperch, $w^{`}$ lf $d^{`} g$ ). The typology can sometimes be refined, as in the case of the last subcategory of hybrid compounds: bull terrier, bullmastiff, and $w^{`}$ If $d^{`} g$ exemplify genetic hybridity, and ape-man, bear cat, and tr`utperch perceptual hybridity (ape-man does not denote the hybrid offspring of an ape and a man, but the "missing link" in the

\footnotetext{
${ }^{14}$ When the two compounding elements denote substances (ex.: gum and resin, $t$ 'xin and antit $x i n$ ), the hybridity test must be modified: "(an) X.Y is a mixture ' $f(a n) X$ and (a) $Y$ ”.
} 
phylogenesis of $H^{`}$ minini; similarly, Ailurus fulgens and Perc`psis ‘misc`maycus are not crossbreeds). Even though they are very scarce in English, three other types of N.N coordinate compounds need to be mentioned as they belong to semantic categories that are well-established in a wide variety of languages. ${ }^{15}$ Collective, hypernymic, and tautological compounds all belong to the additional type, but they differ from the prototype as none passes the standard additionality test. In the case of collective compounds, the test is negative when the compound is in the singular, but positive when it is pluralized: *an Angl`-Sax` $n$ is an Angle plus a Sax`n / Angl`-Sax`ns are Angles plus Sax ${ }^{`}{ }^{16}{ }^{16}$ The addition is only possible between collective entities; in the singular, the compound is semantically disjunctive: “(an) X.Y is either (an) X' $r$ (a) Y”. Gerund-participle, a neologism coined by Huddleston, ${ }^{17}$ is another collective compound, as the following comment shows:

We c`ined this term precisely $f$ $r$ the uni` $n ` f$ what is den`ted by the traditi nal terms gerund and present participle because we d' $n^{\prime} t$ believe the traditi nal distincti` $n$ between them is s`und: in saying that such and such a f $\mathrm{rm}^{\mathrm{r}}$ is a gerund-participle, theref re, we are $n^{`} t$ saying that it is simultane` usly a gerund and a participle, but that it bel`ngs $t$ ` a single categ `ry c`vering $b^{`}$ th traditi nal 'nes. ${ }^{18}$

Hypernymic compounds differ from the prototype in so far as the addition does not simply involve the two compounding elements, but a whole set of co-hyponyms: "(an) $X . Y$ is (an) $X$, (a) $Y$, plus their c`-hyp 'nyms”. M`n-Khmer does not refer only to Mon and Khmer, but to a family of 147 languages including Vietnamese and Nicobarese; similarly, $s^{`} l$-fa refers to the whole set $d$, $r e, m i$, $f a, s^{`} l, l a$, and $t i$. Finally, tautological compounds are those compounds uniting two quasisynonyms, such as c`urtyard, hustle-bustle, and pathway.

\footnotetext{
${ }^{15} \mathrm{Cf}$. Wälchli, 141, 143-6, 151.

${ }^{16}$ The reading of the compound is based on the sense " $a$ member ' $f$ `ne ` $f$ the Germanic pe`ples wh` settled in Britain in the fifth and sixth centuries".

${ }^{17}$ Huddleston, 74-5.

${ }^{18}$ Bauer and Huddleston, 1648.
} 


\section{Adjective-adjective compounds}

A.A coordinate compounds belong to two semantic classes. The overwhelming majority is of the additional type as they pass the test " $t$ ' be $X . Y$ is $t$ ' be $X$ and $t$ ' be $Y$ " (ex.: bittersweet, deaf-blind, deaf-mute, ‘bsessive-c`mpulsive, pale-dry, passive-aggressive). Several additional compounds have specific semantics: manic-depressive is an alternate compound (“alternately $X$ and $Y$ ”), shabbygenteel an adversative compound (“X but $Y$ ”); squiggly-wiggly, teeny-tiny, teeny-weeny, and yummy-scrummy are tautological compounds ( $X=Y$ "). Several compounds are however not additional; it is the case of the six tertiary colours, yell`w-'range, red-'range, red-vi`let, bluevi let, blue-green, and yell w-green, produced by an equal mixture of a primary colour and a secondary colour adjacent to it on the colour wheel, and of the culinary terms medium-rare and medium-well. ${ }^{19}$ These compounds do not pass the additionality test given above, but they pass a hybridity test: " $t$ ' be $X . Y$ is $t$ ' be ab`ut midway between $X$ and $Y$ ".

The semantic interpretation of a compound adjective associating two colours is often delicate. Bauer and Huddleston ${ }^{20}$ consider that blue-grey and 'range-red are semantically right-headed, and therefore subordinate. The two compounds are however not institutionalized, and they may refer to any shade comprised between slightly-blue grey and an equal mixture of blue and grey, between slightly-orange red and an equal mixture of orange and red. As a result, the two readings subordinate and coordinate - coexist. The meaning of a two-colour compound is not univocal, except when semantic subordination is marked morphologically, by the addition of a suffix on the subordinate element (ex.: `rangey-red refers to a shade of red, reddy-`range to a shade of orange; greenish-blue refers to a shade of blue, bluish-green to a shade of green). The interpretative ambiguity is exemplified by the treatment of blue-black in dictionaries. In some, the adjective refers to bluish black:

"Black `r dark with a tinge `f blue.” (OEDO)

"Black with bluish highlights." (RHUD)

\footnotetext{
${ }^{19}$ Well is a clipped form of well-d $n e$.

${ }^{20}$ Bauer and Huddleston, 1658.
} 
"Black tinged with blue ' $r$ with a blue sheen when caught by the light." (EWED)

In others, it refers to dark blue, i.e. a colour which is perceptually about midway between primary blue and black:

"Dark blue." (PED)

"Very dark blue." (AHD4)

"A very dark blue that s`metimes l’ ks blue and s`metimes black." (CALD2)

It is therefore preferable to conclude that there is no clear perceptual boundary between the hybrid coordinate reading and the subordinate reading of a two-colour compound.

\section{Verb-verb compounds}

V.V coordinate compounds belong to three semantic categories: asynchronous compounds, synchronous compounds, and disjunctive compounds. The relation of asynchrony between the compounding elements is identified through the paraphrase " $t$ ' $X$ and then $t$ ' $Y$ ". Asynchronous V.V compounds are either verbs (ex.: $d r^{`} p$-kick, freeze-dry, tie-dye) or adjectives (ex.: $c^{`}{ }^{`} k$-chill, drink-drive, fly-drive, push-pull, read-write, $r^{`} l l$-`$^{\prime} n r^{`} l l-` f f, s t^{`} p$ - $g^{`}, s t^{`} p$-start). ${ }^{21}$ Some of the deverbal compounds - push-pull, st` - $^{\prime} g^{`}$, st $^{`}$ p-start - differ from the prototype as their semantics include an iterative nuance, which makes them alternate compounds (" $t^{\prime} X$ and $t^{\prime} Y$ alternately"). The relation of synchrony is antithetical to that of asynchrony, and is identified through the paraphrase " $t$ ' $X$ and $t^{\prime} Y$ at the same time". Synchronous compounds are either verbs (ex.: sleepwalk, ${ }^{22}$ stir-fry) or adjectives (ex.: win-win, w`rk-study). Disjunctive compounds put together compounding elements which are disjunctively related (" $X{ }^{\prime} r Y^{\prime}$ ). This reading is exemplified by the noun lend-lease and the adjective pass-fail.

\footnotetext{
${ }^{21} C^{`} ` k$-chill, fly-drive, push-pull, and $r^{`} l l$ - $^{\prime} r^{`} l l-` f$ are also institutionalized as deadjectival noun compounds.

${ }^{22}$ The verbal status of the left compounding element is not always obvious, but if the element is institutionalized as a verb, the V.V analysis of the compound seems almost irresistible, as the repeated attestation of the form slept-walked for instance demonstrates: "In Germany, Stuart m rally slept-walked thr ' ugh the Reich, until the saturati $n$ b`mbing disturbed his peace.”, The Blackwell C`mpani $n t^{`} M$ 'dern Irish Culture, edited by W. J. McCormack, 2001, Blackwell, p. 643; "He t'ld us this was very necessary as he slept walked and was afraid he w'uld fall 'ff this table-r`ck and kill himself.”, Al`ng the Way, by B. C. Fincher-Young, 2004, Xlibris, p. 144.
} 


\section{Conclusion}

The aim of this article has been to make substantial progress in the description of the semantics of English coordinate compounds. Ten Hacken, Wälchli, and Bauer ${ }^{23}$ have convincingly tackled the question of classifying coordinate compounds, but their approach is multilingual, and their English examples are too rare. The typology summed up in Table 2 is doubly original: it does not focus solely on nominal compounds, and it brings to the fore the least-known class of English coordinate compounds, that of hybrid compounds, which are relatively scarce, but appear in other languages, as in Romance, where they are attested for the three major lexical classes:

- Spanish: gallipav`<gall “cock” + pav` “peacock” = turkey ${ }^{24}$

- Portuguese: verdissec < verde "green" + sec " "dry" = half-dry

- Italian: d'rmiveglia < d rmire "sleep" + vegliare "be awake" = a state intermediate between sleeping and being awake

Table 2

Semantic typology of English coordinate compounds

\begin{tabular}{|c|c|c|}
\hline \multirow{6}{*}{ N.N } & \multirow{2}{*}{\multicolumn{2}{|c|}{$\frac{\text { multifunctional }}{\text { hybrid }}$}} \\
\hline & & \\
\hline & \multirow{4}{*}{ additional } & prototypical \\
\hline & & collective \\
\hline & & hypernymic \\
\hline & & tautological \\
\hline \multirow{5}{*}{ A.A } & \multicolumn{2}{|c|}{ hybrid } \\
\hline & \multirow{4}{*}{ additional } & prototypical \\
\hline & & alternate \\
\hline & & adversative \\
\hline & & tautological \\
\hline \multirow{4}{*}{ V.V } & \multicolumn{2}{|c|}{ synchronous } \\
\hline & \multirow{2}{*}{ asynchronous } & prototypical \\
\hline & & alternate \\
\hline & \multicolumn{2}{|c|}{ disjunctive } \\
\hline
\end{tabular}

\footnotetext{
${ }^{23}$ Bauer, Dvandva.

${ }^{24}$ In present-day Spanish, pav denotes a turkey, and pav` real a peacock, but gallipav` was coined by the early conquistadores.
} 
A contrastive approach is no doubt the most fruitful path to explore if one wants to delve deeper into the semantic intricacies of coordinate compounding, but this can only be done after gathering a wealth of data in a host of typologically diverse languages, and as this task cannot be automated, this domain will need a lot of collective time and effort to be precisely charted cross-linguistically.

\section{References}

AHD4 = American Heritage Dicti nary, fourth edition. Boston: Houghton Mifflin, 2000. $<$ http://www.bartleby.com/61/>.

Arnaud, Pierre J. L. "Document de travail sur les noms composés anglais.” Unpublished manuscript. Lyon 2 University: English Department, 2002.

Bauer, Laurie. English W`rd-F`rmati`n. Cambridge: Cambridge University Press, 1983.

—-—. A Gl`ssary `fM`rph`l'gy. Edinburgh: Edinburgh University Press, 2004.

- - - "Dvandva". W` rd Structure, forthcoming.

- - - , and Rodney Huddleston. "Lexical Word-formation.” In The Cambridge Grammar 'f the English Language, edited by Rodney Huddleston and Geoffrey K. Pullum, 1621-1721. Cambridge: Cambridge University Press, 2002.

Boisson, Claude. L’Accentuati`n des c`mp`sés en anglais c`ntemp`rain. Avec quelques c`ntributi`ns à l'accent`l'gie générale. PhD dissertation, Paris 7 University. Lille: Atelier National de Reproduction des Thèses, 1980.

CALD2 = Cambridge Advanced Learner's Dicti nary, second edition. Cambridge: Cambridge University Press, 2005. <http://dictionary.cambridge.org/>.

COD10 = C`ncise Oxf $r d$ Dicti`nary, tenth edition. Oxford: Oxford University Press, 1999.

Cruse, D. Alan. Lexical Semantics. Cambridge: Cambridge University Press, 1986. 
EWED = Encarta World English Dictionary. New York: St Martin's Press, 1999. $<\underline{\text { http://encarta.msn.com/encnet/features/dictionary/dictionaryhome.aspx }}>$.

Hacken, Pius ten. Defining Morphology: A Principled Approach to Determining the Boundaries of Compounding, Derivation, and Inflection. Hildesheim: Olms, 1994.

Huddleston, Rodney. “The Verb”. In The Cambridge Grammar of the English Language, edited by Rodney Huddleston and Geoffrey K. Pullum, 74-214. Cambridge: Cambridge University Press, 2002.

Katamba, Francis, and John Stonham. Morphology, second edition. Basingstoke: Palgrave Macmillan, 2006.

OEDO = Oxford English Dictionary Online. Oxford: Oxford University Press. $<$ http://www.oed.com/>.

Olsen, Susan. "Copulative Compounds: A Closer Look at the Interface between Syntax and Morphology". In Yearbook of Morphology 2000, edited by Geert Booij and Jaap van Marle, 279320. Dordrecht: Kluwer, 2001.

PED = Penguin English Dictionary. London: Penguin, 2003.

Plag, Ingo. Word-Formation in English. Cambridge: Cambridge University Press, 2003.

RHUD = Random House Unabridged Dictionary. New York: Random House, 1997. <http://www.infoplease.com/dictionary.html $>$.

Wälchli, Bernhard. Co-Compounds and Natural Coordination. Oxford: Oxford University Press, 2005. 